\title{
Power Minimization for Multi-Cell OFDM Networks Using Distributed Non-cooperative Game Approach
}

\author{
Zhu Han, Zhu Ji, and K. J. Ray Liu \\ Electrical and Computer Engineering Department, University of Maryland, College Park.
}

\begin{abstract}
In this paper, we use noncooperative game approach to have distributed sub-channel assignment, adaptive modulation, and power control for multi-cell OFDM networks. The goal is to minimize the overall transmitted power under each user's maximal power and minimal rate constraints. Our contribution is to model and solve this complicated problem by a distributed noncooperative game approach: Each user water-fills its power to different subchannels regarding other users' powers as interferences. A noncooperative game is constructed for each user to compete with others. A method is constructed as a mediator (judge) for the game. From the simulation results, the proposed scheme reduces the overall transmitted power greatly compared with the fixed channel assignment algorithm and pure water-filling algorithm.
\end{abstract}

\section{INTRODUCTION}

Orthogonal Frequency Division Modulation (OFDM) is a promising modulation scheme for wireless broadband networks. In multiuser OFDM networks, efficient resource allocation can greatly improve system performances by performing sub-channel assignment, rate allocation, and power control for different users.

In multi-cell OFDM systems, the resource allocation problem becomes more complicated, even if the assignment of sub-channels to users is predetermined. This is because users in different cells reuse the same sub-channels and cause interferences to each other. If the number of co-channel users is relatively large, the interference seen by a user in a sub-channel can be approximated by a Gaussian random variable applying the central limit theorem. In this case, water-filling algorithm provides a good solution. When the channel assignment is fixed, many iterative water-filling methods are proposed in [1], [2], [3], [4], [5] to maximize the rate with power constraints. However, if the sub-channel assignment is not predetermined, all possible combinations of co-channel users should be checked to determine the optimal resource allocation. In [6], the authors present heuristic distributed algorithms, which are based on iterative water-filling with removing sub-channels of low signal to interferences plus noise ratio (SINR).

In the multi-cell systems, the mobile users do not have the knowledge of other users conditions and cannot cooperate with each other, they act selfishly to maximize their own performances in a distributed way. Such a fact motivates us to adopt the game theory [8]. The resource allocation can be modelled as a noncooperative game that deals largely with how rational and intelligent individuals interact with each other in an effort to achieve their own goals. In the resource allocation game, each mobile user is self-interested and trying to optimize his utility function, where the utility function represents the user's performance and controls the outcomes of the game.
In this paper, we want to minimize the overall transmitted power under each user's maximal power and minimal rate constraints. By noncooperative game theory approach, we find the following facts: If the co-channel interferences are small, users can share the sub-channels for transmission. In this case, by carefully designing the utility function, the noncooperative game for each user to compete the resources will be balanced in an optimal and unique Nash equilibrium point (NEP). If the co-channel interferences are severe for some sub-channels, NEP may not be optimal and there might be multiple NEPs. In order to deal with this situation, some users with bad channels or large interferences to others must be kicked out from using these sub-channels, so that the rest of the users can make good use of the corresponding sub-channels. We design the utility function for each user, define the criterion as a game rule to kick out users, and develop an adaptive algorithm for resource allocation. From the simulation results, we can see that the proposed scheme can reduce the overall transmitted power greatly compared to the fixed channel assignment algorithm and pure water-filling algorithm.

The rest of this paper is organized as follows: In Section II, we give the system model and formulate the problem. In Section III, the adaptive algorithm of the noncooperative approach is developed. In Section IV, we show the simulation results. In Section V, conclusions are drawn.

\section{System Model And Problem Formulation}

The $K$ co-channel cells are taken into consideration that may exist in distinct cells of OFDM networks. Each cell consists of a mobile user and its assigned base station. Assume coherent detection is possible so that it is sufficient to model this multiuser system by an equivalent baseband model. We also assume the different links among cells are synchronized. ${ }^{1}$ The total number of OFDM sub-channels is $L$. For the uplink case, the sampled signal on the $l^{\text {th }}$ sub-channel of the $i^{\text {th }}$ user can be expressed as:

$$
x_{i}^{l}(n)=\sum_{k=1}^{K} \sqrt{P_{k}^{l} G_{k i}^{l}} s_{k}^{l}(n)+n_{i}^{l}(n)
$$

where $P_{k}^{l}$ and $G_{k i}^{l}$ is the transmitted power and propagation loss from the $k^{\text {th }}$ user to the $i^{\text {th }}$ base station in the $l^{\text {th }}$ sub-channel, respectively, $s_{k}^{l}$ is message symbol from the $k^{t h}$ user to the $i^{\text {th }}$ base station at time $n$, and $n_{i}^{l}(n)$ is the sampled thermal noise. We assume that the channels change slowly. Without loss of generality, we assume $N_{i}^{l}=E\left(\left\|n_{i}^{l}\right\|^{2}\right)=N_{0}$. The $i^{t h}$ user's SINR at sub-channel $l$ can be expressed as:

\footnotetext{
${ }^{1}$ this assumption can be relaxed using the same approach in this paper.
} 


$$
\Gamma_{i}^{l}=\frac{P_{i}^{l} G_{i i}^{l}}{\sum_{k \neq i} P_{k}^{l} G_{k i}^{l}+N_{0}} .
$$

Rate adaptation such as adaptive modulation provides each sub-channel with the ability to match the effective bit rates, according to the interference and channel conditions. MQAM is a modulation method with high spectrum efficiency. In [7], for a desired rate $r_{i}^{l}$ of MQAM, the BER of the $l^{t h}$ sub-channel of the $i^{t h}$ user can be approximated as a function of the received SINR $\Gamma_{i}^{l}$ by:

$$
\mathrm{BER}_{i}^{l} \approx c_{1} e^{-c_{2} \frac{\Gamma_{i}^{l}}{2^{r_{i}^{l}}}}
$$

where $c_{1} \approx 0.2$ and $c_{2} \approx 1.5$ with small $\mathrm{BER}_{i}^{l}$. Rearrange (3), for a specific desired $\mathrm{BER}_{i}^{l}$, the $i^{\text {th }}$ user's transmission rate of the $l^{t h}$ sub-channel for the SINR $\Gamma_{i}^{l}$ and the desired $\mathrm{BER}_{i}^{l}$ can be expressed as: ${ }^{2}$

$$
r_{i}^{l}=W \log _{2}\left(1+c_{3}^{i} \Gamma_{i}^{l}\right)
$$

where $W$ is the bandwidth and $c_{3}^{i}=-\frac{c_{2}^{i}}{\ln \left(\mathrm{BER}_{i}^{l} / c_{1}^{i}\right)}$. In this paper, for simplicity, we assume all the sub-channels and users have the same BER requirement, i.e., $\mathrm{BER}_{i}^{l}=$ BER, $\forall i, l$.

Each user requires the rate $R_{i}$ and distributed its rate into $L$ sub-channels, i.e., $\sum_{l=1}^{L} r_{i}^{l} \geq R_{i}$. Define rate allocation matrix $[\mathbf{r}]_{i l}=r_{i}^{l}$. Each user's transmitted power is bounded by $P_{\max }$. Define the $K \times L$ channel assignment matrix $\mathbf{A}$ with $[\mathbf{A}]_{i l}=1$, if $r_{i}^{l}>0 ;[\mathbf{A}]_{i l}=0$, otherwise. Therefore, our objective is to minimize the overall transmitted power under the minimal rate and maximal power constraints, i.e.,

$$
\begin{gathered}
\min _{\mathbf{A}, \mathbf{r}} f(\mathbf{r})=\sum_{i=1}^{K} \sum_{l=1}^{L} P_{i}^{l} \\
\text { s.t. }\left\{\begin{array}{l}
\sum_{l=1}^{L} r_{i}^{l}-R_{i} \geq 0, \forall i, \\
\sum_{l=1}^{L} P_{i}^{l}-P_{\max } \leq 0, \forall i, \\
r_{i}^{l}, P_{i}^{l} \geq 0, \forall i, l .
\end{array}\right.
\end{gathered}
$$

Since power is continuous increasing function of rate, the optimum occurs when $\sum_{l=1}^{L} r_{i}^{l}=R_{i}$. The problem in (5) is very difficult to solve by centralized constrained nonlinear integer optimization, because the complexity and communication overhead grows fast as the number of users increases. This motivates us to develop a distributed algorithm with limited controls by using the game theory approach.

\section{Noncooperative Game Approach}

In this section, our focus is to solve (5) by noncooperative game theory. First, we analyze the system feasible region. Then we will construct the game. A two-user two-subchannel example is given to show insights. The properties of the NEP are analyzed. Finally, an iterative algorithm for multiple users with a game mediator is developed.

\footnotetext{
${ }^{2}$ Here the rate of MQAM is assumed to be continuous. Discrete MQAM can be applied in a similar way for the approach developed later in this paper.
}

\section{A. System Feasibility Region}

In order to ensure the desired BER, for every subchannel, every user should have SINR no less than the required SINR $\gamma_{i}^{l}$, i.e., $\Gamma_{i}^{l} \geq \gamma_{i}^{l}, \forall i, l$. Rewrite these inequalities in matrix form, we have

$$
\left(\mathbf{I}-\mathbf{D}^{l} \mathbf{F}^{l}\right) \mathbf{P}^{l} \geq \mathbf{v}^{l}, \forall l,
$$

where $\mathbf{I}$ is a $K \times K$ identity matrix, $\mathbf{v}^{l}=\left[v_{1}^{l}, \ldots, v_{K}^{l}\right]^{\prime}$ with $v_{i}^{l}=N_{0} \gamma_{i}^{l} / G_{i i}, \mathbf{D}^{l}=\operatorname{diag}\left\{\gamma_{1}^{l}, \ldots, \gamma_{K}^{l}\right\}$, and

$$
\left[\mathbf{F}_{i j}^{l}\right]= \begin{cases}0 & \text { if } j=i, \\ \frac{G_{j i}^{l}}{G_{i i}^{l}} & \text { if } j \neq i .\end{cases}
$$

By Perron-Frobenius theorem, there exists a positive power allocation if and only if the maximum eigenvalue of $\mathbf{D}^{l} \mathbf{F}^{l}$, i.e. spectrum radius $\rho\left(\mathbf{D}^{l} \mathbf{F}^{l}\right)$, is inside unit circle. When $\left|\rho\left(\mathbf{D}^{l} \mathbf{F}^{l}\right)\right|<1$, the optimal power solution is

$$
\mathbf{P}^{l}= \begin{cases}\left(\mathbf{I}-\mathbf{D}^{l} \mathbf{F}^{l}\right)^{-1} \mathbf{v}^{l}, & \left|\rho\left(\mathbf{D}^{l} \mathbf{F}^{l}\right)\right|<1 \\ +\infty, & \text { otherwise }\end{cases}
$$

The system feasibility region $\Omega$ is defined as the supporting domain where there exist solutions and power constraint in (5) is satisfied. The condition for (7) to have finite solutions is a necessary condition for existence of feasible $\Omega$ and convergence of the algorithm proposed later.

\section{B. Noncooperative Game and Nash Equilibrium}

Each user wants to minimize its transmitted power by allocating its rate into the different sub-channels, regardless other users in a distributed way. Define $\mathbf{r}_{i}=\left[r_{i}^{1} \ldots r_{i}^{L}\right]^{T}$, the noncooperative game can be written as:

$$
\text { Game: } \arg \min _{\mathbf{r}_{i} \in \Omega} u_{i}=\sum_{l=1}^{L} P_{i}^{l} \text {, s.t. } \sum_{l=1}^{L} r_{i}^{l}=R_{i},
$$

where $u_{i}$ is the utility function defined as the $i^{t h}$ user's transmit power. If the interferences from others are fixed, it is a water filling problem. Define

the solution is

$$
I_{i}^{l}=\frac{\sum_{k \neq i} P_{k}^{l} G_{k i}^{l}+N_{0}}{c_{3}^{i} G_{i i}^{l}}
$$

$$
P_{i}^{l}=\left(\mu_{i}-I_{i}^{l}\right)^{+} \text {and } r_{i}^{l}=\log _{2}\left(1+\frac{P_{i}^{l}}{I_{i}^{l}}\right)
$$

where $y^{+}=\max (y, 0) . \mu_{i}$ is solved by bisection search of

$$
\sum_{l=1}^{L} \log _{2}\left(1+\frac{\left(\mu_{i}-I_{i}^{l}\right)^{+}}{I_{i}^{l}}\right)=R_{i} .
$$

However the interferences from other users do change. Based on the game theory [8], the system will be balanced in a Nash equilibrium defined as:

Definition 1: Define $\mathbf{r}_{i}^{-1}=\left[\mathbf{r}_{1} \ldots \mathbf{r}_{i-1} \mathbf{r}_{i+1} \ldots \mathbf{r}_{L}\right]$. Nash Equilibrium Point $\mathbf{r}_{i}$ is defined as:

$$
u_{i}\left(\mathbf{r}_{i}, \mathbf{r}_{i}^{-1}\right) \leq u_{i}\left(\tilde{\mathbf{r}}_{i}, \mathbf{r}_{i}^{-1}\right), \forall i, \forall \tilde{\mathbf{r}}_{i} \in \Omega, \mathbf{r}_{i}^{-1} \in \Omega^{L-1} .
$$

i.e., given the other users' rate allocation, no user can reduce its transmitted power alone by changing its rate allocation to different sub-channels. 


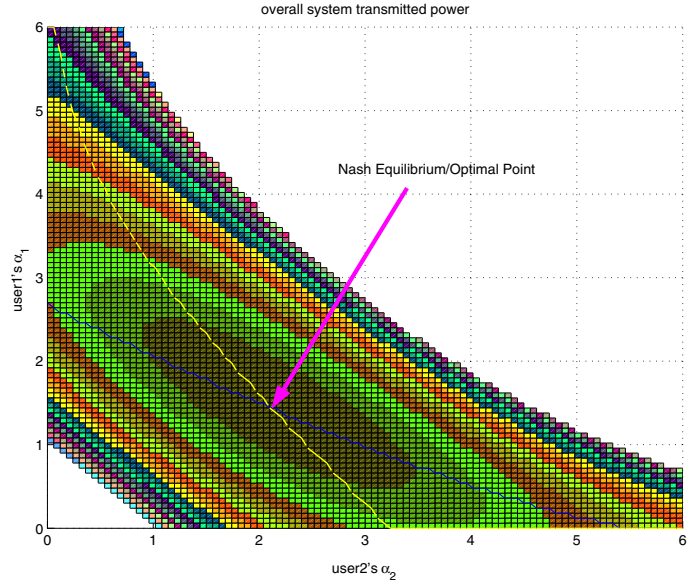

Fig. 1. Two-user example: Unique Optimal NEP

\section{Two-User Two-Sub-channel Analysis}

In order to explain the Nash equilibrium and show the idea of our noncooperative game approach, we analyze a two-user two-sub-channel case in this subsection. Suppose the $i^{t h}$ user puts $\alpha_{i}$ proportion of its rate $R_{i}$ to the first subchannel. The second sub-channel has the rate of $\left(1-\alpha_{i}\right) R_{i}$. Obviously $0 \leq \alpha_{i} \leq 1$. Let $P_{i}^{j}\left(\alpha_{i}\right)$ denote the transmission power of the $i^{\text {th }}$ user on the $j^{\text {th }}$ sub-channel, which is a function of $\alpha_{i}, \forall i$. For simplicity, we write $P_{i}^{j}\left(\alpha_{i}\right)$ as $P_{i}^{j}$. The Lagrangian function of the optimal allocation problem can be represented as

$J\left(\alpha_{1}, \alpha_{2}\right)=P_{1}^{1}+P_{1}^{2}+P_{2}^{1}+P_{2}^{2}+\sum_{i=1}^{2} \mu_{i} \alpha_{i}+\sum_{i=1}^{2} \lambda_{i}\left(1-\alpha_{i}\right)$

where $\mu_{i}$ and $\lambda_{i}$ are the Lagrange multipliers. By taking derivatives of $\alpha_{1}$ and $\alpha_{2}, \frac{\partial J\left(\alpha_{1}\right)}{\partial \alpha_{1}}=0 ; \quad \frac{\partial J\left(\alpha_{2}\right)}{\partial \alpha_{2}}=0$, we can obtain the global optimal solution. However, global channel information such as $G_{k i}, k \neq i$ is necessary to solve (13), which causes great implementation difficulty in multicell OFDM networks.

On the other hand, for Nash equilibriums, the users perform the water-filling algorithms in a distributed way by using local information only. The Lagrangian functions of optimization problems are illustrated as

$$
\begin{aligned}
& J_{1}=P_{1}^{1}+P_{1}^{2}+\mu_{1}^{\prime} \alpha_{1}+\lambda_{1}^{\prime}\left(1-\alpha_{1}\right), \\
& J_{2}=P_{2}^{1}+P_{2}^{2}+\mu_{2}^{\prime} \alpha_{2}+\lambda_{2}^{\prime}\left(1-\alpha_{2}\right),
\end{aligned}
$$

where $\mu_{i}^{\prime}$ and $\lambda_{i}^{\prime}$ are the Lagrange multipliers. The Nash equilibriums of the above channel allocation problem can be obtained by solving $\frac{\partial J_{1}\left(\alpha_{1}\right)}{\partial \alpha_{1}}=0 ; \quad \frac{\partial J_{2}\left(\alpha_{2}\right)}{\partial \alpha_{2}}=0$. Obviously, the solutions of global optimum and Nash equilibrium are different. However from the observation, the two solutions are close when the minimal rates are low.

In order to compare the Nash equilibriums and the optimal solution, a simple two-user two-sub-channel example is illustrated as follows. The simulation setup is: $\mathrm{BER}=10^{-3}, N_{0}=10^{-3}, P_{\max }=10^{4}$, and

$$
G^{1}=\left[\begin{array}{ll}
0.0631 & 0.0100 \\
0.0026 & 0.2120
\end{array}\right], G^{2}=\left[\begin{array}{ll}
0.4984 & 0.0067 \\
0.0029 & 0.9580
\end{array}\right] .
$$

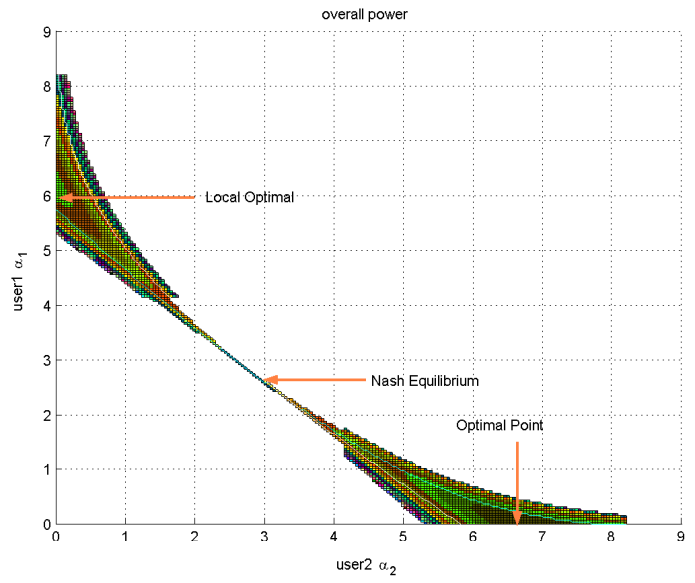

Fig. 2. Two-user example: Multiple Local Optima

Fig. 1 shows the overall power contour as a function of two users' rate allocations, where $R_{1}=R_{2}=6$. The axes are $\alpha_{1}$ and $\alpha_{2}$, respectively. The two curves show the minimal locations for the two users' own powers when the interference from the other user is fixed, respectively. Each user tries to minimize its power by adjusting its rate allocation so that the operating point is more close to the curve. Consequently, the cross is a Nash equilibrium, where no user can reduce its power alone. We can see that the Nash equilibrium under this setup is unique and optimal for the overall power. (It is worthy to mention that the feasible domain is not convex at all.) Fig. 2 shows the situation when $R_{1}=R_{2}=8$. Because the rate is increased, the co-channel interferences are increased and the NEP is no longer the optimum. There exists more than one local optima and the global optimum occurs when user1 doesn't occupy the sub-channel 1. Fig. 3 shows the situation when $R_{1}=R_{2}=8.5$. The contour graph is not connected. There are two NEPs and two local optima. Under the above two conditions, we need to remove users from using the subchannels. If we further increase $R_{1}=R_{2}=10$, there exists no feasible area, i.e., both users cannot have a resource allocation that satisfies both power and rate constraints. In this case, the minimal rate requirement should be reduced.

From the above observations, we can see that the behaviors of the optimal solution and NEP depend on how severe interferences are. In order to let NEP converge to the desired solution, we need to find a criterion as the game rule to decide whether the users can make a good use of the sub-channels like the situation in Fig. 1. If not, we should decide which user should be kicked out from using the sub-channels. The following two theorems are proved for the properties of NEP.

\section{Properties of Nash Equilibrium}

Theorem 1: There exists NEP in the proposed game defined in (8), if $\Omega$ is not empty.

Proof: In [8], it has been shown a NEP exists, if $\forall i$

1. $\Omega$, the support domain of $u_{i}\left(\mathbf{r}_{i}\right)$, is a nonempty, convex, and compact subset of some Euclidean space $\Re^{L}$.

2. $u_{i}\left(\mathbf{r}_{i}\right)$ is continuous in $\mathbf{r}_{i}$ and quasiconvex in $r_{i}^{l}$. 


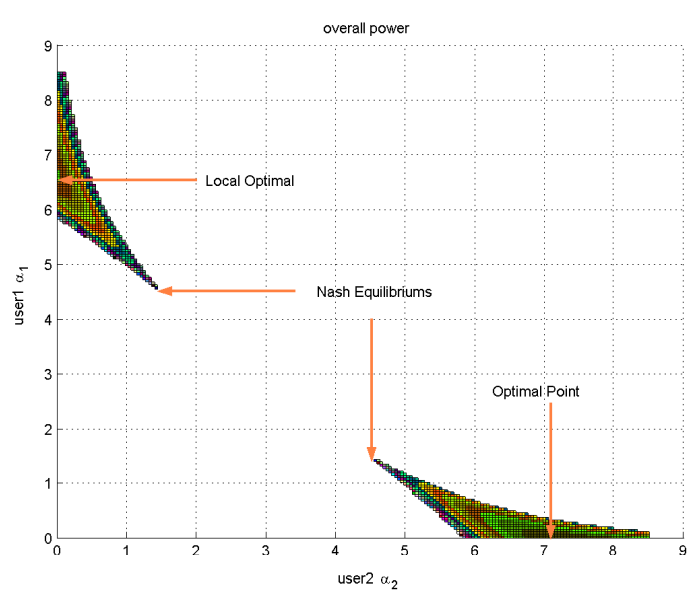

Fig. 3. Two-user example: Multiple NEPs

We consider that each user allocates its transmitted power to different subchannnels first. Since each subchannel can be allocated by $P_{\max }$ and overall transmitted power for all sub-channels is linearly constraint by $P_{\max }$, the supporting domain for power allocation is compact and convex. Because rate is a linear function of transmitted power if the interferences are fixed, the supporting domain $\Omega$ for $r_{i}^{l}, \forall l$ is a convex and compact subset of some Euclidean space $\left(\Re^{+}\right)^{L}$. It is worthy mentioning that $\Omega^{K}$ is not convex and one example is shown in Fig. 1. But our proof only needs that $\Omega$ is convex and nonempty.

From (2) and (4), when the watterfilling is done for (8),

$$
u_{i}=\sum_{l=1}^{L}\left(\frac{\left(2^{r_{i}^{l}}-1\right)\left(\sum_{k \neq i} P_{k}^{l} G_{k i}^{l}+N_{0}\right)}{c_{3}^{i} G_{i i}^{l}}-\mu_{i} r_{i}^{l}\right) .
$$

Obviously, it is continuous and convex for $\mathbf{r}_{i}$. QED

Theorem 2: If the global minimum of (5) occurs when $r_{i}^{l}>0, \forall A_{i l} \neq 0$ and $\sum_{l=1}^{L} P_{i}^{l}<P_{\max }$ and $\sum_{l=1}^{L} r_{i}^{l}=$ $R_{i}, \forall i$, the NEP satisfies the necessary Karush-KuhnTucker (KKT) condition [9].

Proof: $\quad$ First, if $\sum_{l=1}^{L} P_{i}^{l}<P_{\max }$ and $\sum_{l=1}^{L} r_{i}^{l}=$ $R_{i}, \forall i$ at NEP, the iterative water-filling converges. For each user, the resource allocation is optimal if the interferences are considered as noises. By Lagrangian method, define $\nabla=\frac{\partial}{\partial \mathbf{r}_{i}}$, the following equation hold at the NEP when power is less than $P_{\max }$.

$$
\nabla\left(\sum_{l=1}^{L} P_{i}^{l}\right)-\mu_{i} \nabla\left(\sum_{l=1}^{L} r_{i}^{l}-R_{i}\right)=0 .
$$

For the problem in (5), if $r_{i}^{l}>0, \forall A_{i l} \neq 0$ and $\sum_{l=1}^{L} P_{i}^{l}<$ $P_{\text {max }}, \forall i$, the global optima will satisfy the KKT condition without considering the inequality constraints:

$$
\sum_{i=1}^{K} \nabla\left(\sum_{l=1}^{L} P_{i}^{l}\right)-\sum_{i=1}^{K} \mu_{i} \nabla\left(\sum_{l=1}^{L} r_{i}^{l}-R_{i}\right)=0 .
$$

Obviously, when the iterative water-filling converges, (18) will be satisfied from (17). So the KKT necessary condition is satisfied for NEP.

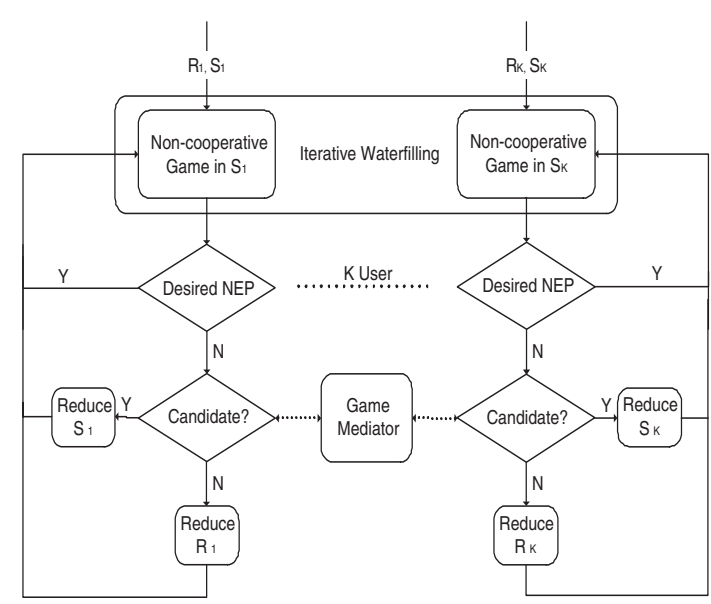

Fig. 4. Multiuser Noncooperative Game

\section{E. Distributed Power Minimization Algorithm}

Before developing the proposed algorithm, we analyze two extreme cases. In the first case, the groups of subchannels are assigned to different cells without overlapping such that there are no co-channel interferences among cells. We call it the fixed channel assignment scheme. However, this extreme method has the disadvantages of low spectrum efficiency because of the low frequency re-usage. In the second extreme case, all the users share all the sub-channels. We call it pure water-filling scheme. From Fig. 2 and Fig. 3 , we can see that the system can be balanced at the undesired point, because of the severe inter-cell co-channel interferences. So the facts motive us to believe that the optimal resource allocation is between these two extreme cases, i.e., each sub-channel can be shared by only a group of selected users for transmission.

The block diagram of the proposed algorithm is shown in Fig. 4. We define the sub-channel set that the $i^{\text {th }}$ user can allocate their rates as transmission group $S_{i}$. Each user plays the noncooperative game to minimize its power. If the game cannot converge to a good solution, a mediator is introduced to kick out some users from using the sub-channel. If no candidate can be removed, the required rate has to be reduced. Here, we assume that base stations can accurately measure the channel gains of their associated mobile users. Moreover, the feedback channel exists from the base station to the mobile user. The proposed distributed algorithm is shown in Table I. First, we initialize the channel and power allocation so that the constraints are satisfied, which ensure the initial point located in the feasible set. This can be obtained by applying any heuristics or greedy approaches. Then, each user minimizes its own utility function, i.e. transmitted power, in a distributed game by applying water-filling. The system will be balanced at some NEP.

If the co-channel interferences are not large, the NEP should be the desired solution. Three criteria are used in the proposed algorithm to judge if the NEP is desired: 1) If the constraints of minimal rate and maximal transmitted power are satisfied; 2) If the NEP is not a local optimum; 3 ) 
Table I: Distributed Power Minimization Algorithm

\begin{tabular}{l} 
1. Initialization: \\
The sub-channels are assigned to the users \\
while satisfying the power and rate constraints. \\
\hline 2. Water Filling: \\
each user have noncooperative game in (8). \\
3. Desired NEP: \\
if $\sum_{l=1}^{L} P_{i}^{l}<P_{\text {max }}$ and $\sum_{l=1}^{L} r_{i}^{l}=R_{i}$ and \\
if not local minimum on boundary and \\
if each user's power is non-increasing, go to Step $2 ;$ \\
4. Sub-channel Removal/Rate Reduction: \\
remove sub-channel from transmission group by $(19)$ \\
go to Step 2. If no user can reduce his transmission \\
group, reduce $R_{i}$, go to Step 2 .
\end{tabular}

If transmit power is decreasing during NEP convergence. From Theorem 1, Theorem 2, and the previous observations, if any of these criteria are not satisfied, the system is probably balanced at an undesired solution. So a game mediator needs to redefine the game by reducing the number of users that share the sub-channels, i.e., changing $S_{i}$.

The criterion to decide which user to be removed from using which sub-channel is determined by the SINR level within transmission groups as

$$
(l, j)=\arg \min _{l, j} \frac{P_{j}^{l} G_{j j}^{l}}{\sum_{k \neq j} P_{k}^{l} G_{k j}^{l}+N_{0}} .
$$

Because of the minimal rate requirement, the selected user might not be able to be removed. Then the algorithm tries to select the next candidate in (19). The criterion for whether or not the user can be removed from the transmission group is determined by three factors: 1) Each user must has at least one sub-channel to transmit. 2) No subchannel is wasted, i.e., at least one user is assigned for each sub-channel. 3) User cannot be kicked out from the subchannel, if the user cannot transmit his rate $R_{i}$ using the rest of sub-channels, even though he occupies them alone. If no user can be removed from the transmission group, the minimal rate $R_{i}$ must be reduced. This situation happens when the system is very crowed and the co-channel interference is large.

\section{Simulation Results}

To show the improvements of the proposed algorithm, we set up two simulations consisting of a two-cell case and a seven-cell case. The base station is located at the center of each cell and one co-channel mobile per cell is generated as a uniform distribution within the corresponding cell for each simulation instance. The propagation model takes into consideration of path loss and shadowing. The received signal (in $\mathrm{dB}$ ) at distance $d$ from the base station is $L(d)=L\left(d_{0}\right)+10 \alpha \log _{10} \frac{d}{d_{0}}$, where $d_{0}=10 \mathrm{~m}$ is used as a reference point in measurements $\left(L\left(d_{0}\right)=0 \mathrm{~dB}\right)$ and $\alpha$ is set to 3.5. Shadow fading for each user is modelled as an independent log-normal random variable with standard deviation $\sigma=10 \mathrm{~dB}$. The four-path Rayleigh model is taken into consideration to simulate the frequency selective fading channels, which has an exponential power profile with

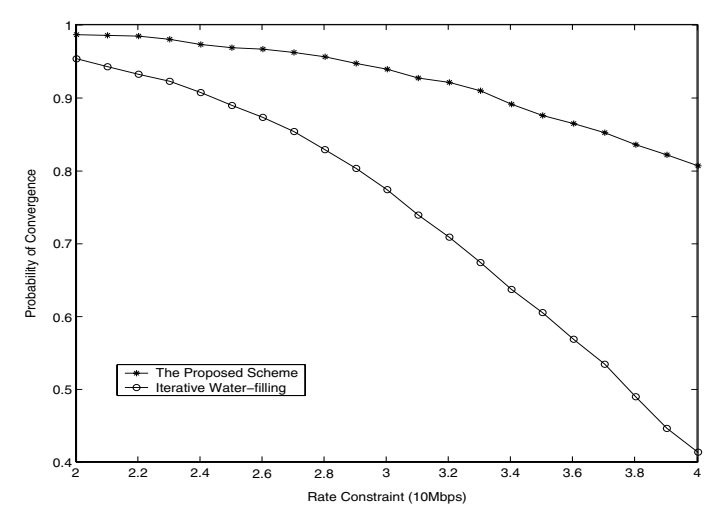

Fig. 5. Convergence Probability vs. Rate Constraint

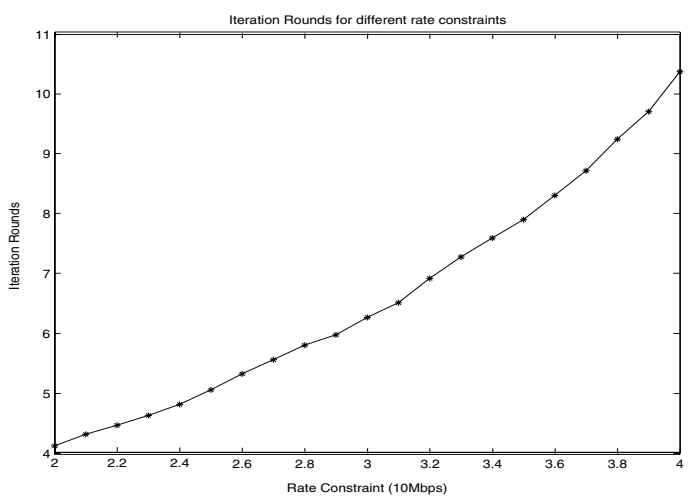

Fig. 6. Convergence Round vs. Rate Constraint

100ns root-mean-square (RMS) delay spread. We consider a multi-cell OFDM system with 32 sub-channels in total. The overall bandwidth is $6.4 \mathrm{MHz}$. The receiver thermal noise is $-70 \mathrm{dBm}$. The required BER of the transmitted symbols is $10^{-3}$ for every sub-channel and user, which corresponds to $c_{3}=0.2831$. We define the reuse factor $R_{u}$ as the distance between two base stations $D$ over the cell radius $r$ which is set as $100 \mathrm{~m}$, which is one of the main factors to affect the severeness of co-channel interference. To evaluate the performances, we simulate $10^{3}$ sets of frequency selective fading channels.

First, we show the simulation results for the two-cell case. Let $R_{u}=2$ and assume $R_{i}=R_{j}, \forall i, j$. In Fig. 5, we compare the probability of convergence to the desired NEP of the proposed algorithm with that of the pure water-filling algorithm. It shows that the proposed approach achieves a much higher convergence probability especially with high rate constraint. The reason is because of the proposed user removal mechanism, which ensures that each sub-channel is well utilized. Note that if the convergence cannot be achieved, we decrease the rate constraint till convergence. If Fig. 6, the number of iteration rounds is plotted vs. the rate constraint. We can see that the convergence speed is fast for all rates in the range. More rounds are necessary with larger interferences (higher rate constraint).

In Fig. 7, we show the total transmitted power as the rate constraint $R_{i}$ increases. Compared with the fixed assignment algorithm, the proposed algorithm reduces about $80 \%$ transmission power. This is because the fixed assignment algorithm wastes too many resources by letting only one user occupy one sub-channel. Compared with 


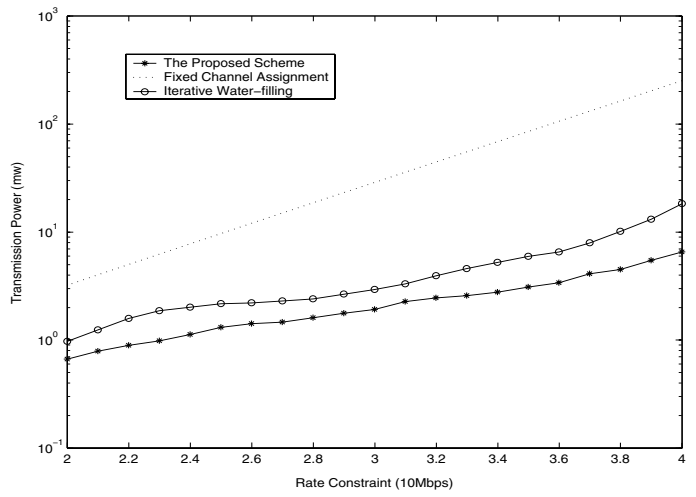

Fig. 7. Total Power vs. Rate Constraint

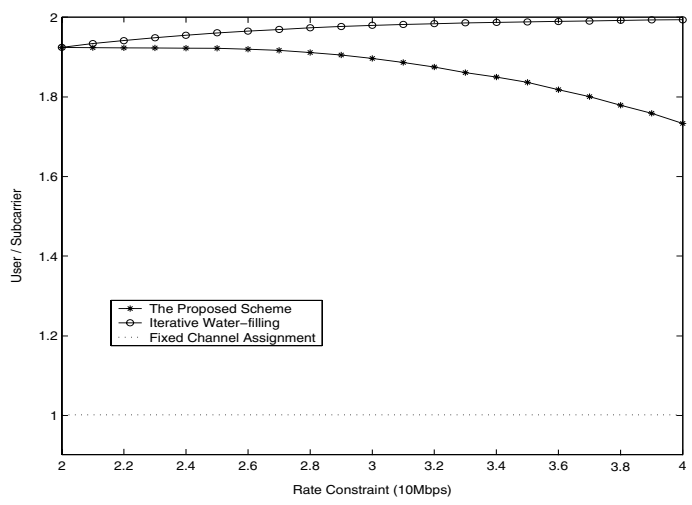

Fig. 8. User per Channel vs. Rate Constraint

the pure water-filling algorithm, the proposed algorithm reduces about 25\% transmission power. This is because pure water-filling algorithm makes some sub-channels overcrowed.

In Fig. 8, we show the number of users occupying each sub-channel when increasing the rate constraint. The fixed channel assignment algorithm always has only one user per channel (UPC). The proposed algorithm has lower UPC compared with the pure water-filling algorithm. For pure water-filling algorithm, some sub-channels may not be allocated any power when the rate constraint is small, because of the low water-filling level. For the proposed algorithm, more users are kicked out from using certain sub-channels when the rate constraint becomes large.

The simulation results for 7-cell networks are shown as follows. The rate constraint is set as 10Mbits for each user. In Fig. 9 and Fig. 10, the total transmission power and UPC are compared between the proposed scheme and the pure water-filling algorithm as the reuse factor increases, respectively. We can see that the proposed algorithm can reduce the overall power about $90 \%$ when the co-channel interferences are severe $\left(R_{u}=2\right)$, which will greatly improve the system performance. Also, the proposed scheme kicks more users out and reduces the number of users per sub-channel for smaller reuse factor. When $R_{u}$ increases, the co-channel interferences reduce. Consequently, two schemes shows similar performances.

\section{Conclusions}

In this paper, the goal is power minimization under the constraints of minimal rate and maximal transmitted

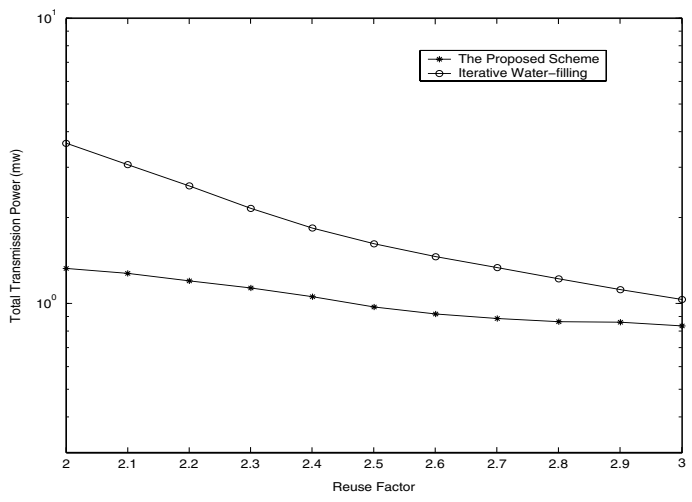

Fig. 9. Overall Power vs. $R_{u}$ for Multicell Case

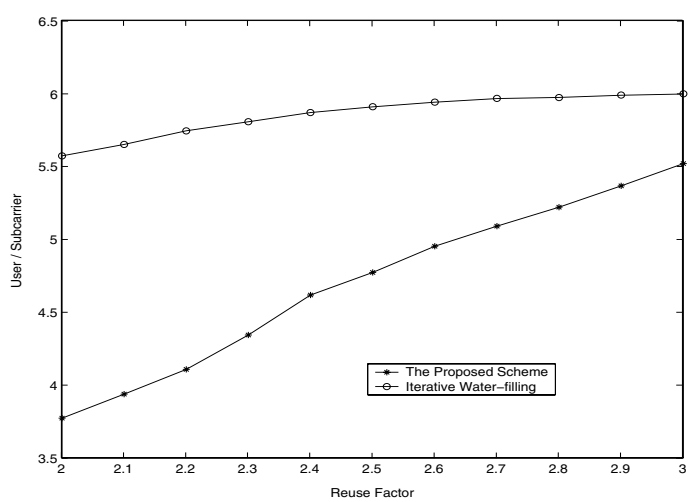

Fig. 10. User per sub-channel vs. $R_{u}$ for Multicell Case

power in multi-cell OFDM systems. We develop a distributed game theory approach to adaptively assign the sub-channels, rate, and powers. From the simulation results, the proposed distributed algorithm reduces the overall transmitted power up to $80 \%$ compared with the fixed assignment scheme for two-cell case, and up to $90 \%$ compared with the pure water-filling scheme for seven-cell case when the co-channel interferences are severe. As a result, the system performances can be greatly improved.

\section{REFERENCES}

[1] W. Yu, G. Ginis, and J.M. Cioffi, "Distributed multiuser power control for digital subscriber lines", IEEE Journal on Sel. Areas in Commun., vol.20, no.5, pp.1105-1114, Jun. 2002.

[2] W. Yu, W. Rhee, S. Boyd, and J.M. Cioffi, "Iterative water-filling for gaussian vector multiple access channels", to appear in IEEE Transactions on Information Theory.

[3] S.T. Chung, S.J. Kim and J.M. Cioffi, "A game-theoretic approach to power allocation in frequency-selective Gaussian interference channels", ISIT '03.

[4] P. Viswanath, D. Tse, and V. Anantharam, "Asymptotically optimal waterfilling in vector multiple access channels", IEEE Transactions on Information Theory, vol.12, no.4, pp.241 267, Jan. 2001.

[5] O. Kaya and S. Ulukus, "Optimum power control for fading CDMA with deterministic sequences", 40th Annual Allerton Conference on Communications, Control and Computing, Oct. 2002.

[6] H. J. Su and E. Geraniotis, "A distributed power allocation algorithm with adaptive modulation for multi-cell OFDM sytems," 5th IEEE International Symposium on Spread Spectrum Techniques and Applications, vol. 2, pp. 474-478, 1998.

[7] S.T. Chung and A.J. Goldsmith, "Degrees of freedom in adaptive modulation: a unified view", IEEE Trans. Commun., vol.49, pp.1561-1571, Sep. 2001.

[8] D. Fudenberg and J. Tirole, Game theory, MIT Press, Cambridge, MA, 1991.

[9] M.S. Barzaraa, Nonlinear programming: theory and algorithms, 2nd ed., John Wiley \& Sons, 1993. 